\title{
More on the Unified Mittag-Leffler Function
}

\author{
Chahnyong Jung ${ }^{1,+}+\mathbb{D}$, Ghulam Farid ${ }^{2,+}$, Hafsa Yasmeen ${ }^{2,+}$ and Kamsing Nonlaopon ${ }^{3, *,+}$ (D) \\ 1 Department of Business Administration, Gyeongsang National University, Jinju 52828, Korea; \\ bb5734@gnu.ac.kr \\ 2 Department of Mathematics, COMSATS University Islamabad, Attock Campus, Attock 43600, Pakistan; \\ faridphdsms@hotmail.com (G.F.); hafsatariq467@gmail.com (H.Y.) \\ 3 Department of Mathematics, Faculty of Science, Khon Kaen University, Khon Kaen 40002, Thailand \\ * Correspondence: nkamsi@kku.ac.th; Tel.: +66-8-6642-1582 \\ + These authors contributed equally to this work.
}

Citation: Jung, C.; Farid, G.;

Yasmeen, H.; Nonlaopon, K. More on the Unified Mittag-Leffler Function. Symmetry 2022, 14, 523. https:// doi.org/10.3390/sym14030523

Academic Editors: Hwa joon Kim and Lorentz Jäntschi

Received: 24 January 2022

Accepted: 1 March 2022

Published: 3 March 2022

Publisher's Note: MDPI stays neutral with regard to jurisdictional claims in published maps and institutional affiliations.

Copyright: (C) 2022 by the authors. Licensee MDPI, Basel, Switzerland. This article is an open access article distributed under the terms and conditions of the Creative Commons Attribution (CC BY) license (https:// creativecommons.org/licenses/by/ $4.0 /)$.

\begin{abstract}
Symmetry is a fascinating property of numerous mathematical notions. In mathematical analysis a function $f:[a, b] \rightarrow \mathbf{R}$ symmetric about $\frac{a+b}{2}$ satisfies the equation $f(a+b-x)=f(x)$. In this paper, we investigate the relationship of unified Mittag-Leffler function with some known special functions. We have obtained some integral transforms of unified Mittag-Leffler function in terms of Wright generalized function. We also established a recurrence relation along with another important result. Furthermore, we give formulas of Riemann-Liouville fractional integrals and fractional integrals containing unified Mittag-Leffler function for symmetric functions.
\end{abstract}

Keywords: unified Mittag-Leffler function; generalized hypergeometric function; Fox's H-function; Wright generalized hypergeometric function; Mellin transform

\section{Introduction and Preliminary Results}

Differentiation operator is known to all of the mathematicians using elementary calculus. For a function $f$, nth derivative of $f$ written as $D^{n} f(x)=d^{n} f(x) / d x^{n}$ is well defined if $n$ is a positive integer. A deep question raised by L'Hospital in 1695 to Leibniz for ascribing $D^{n} f$ meaning, provided $n$ were fraction, drawn attention of the top leading scientists. Since then, a large volume of work is devoted on the applications of the fractional calculus on a variety of differential equations. This led to a huge scientific literature on the use of fractional calculus in fields of science and engineering. These include electromagnetics, fluid flow, viscoelasticity, electrical networks, signals processing, electromagnetic theory, and probability. After appearing as a powerful tool in the development of pure and applied mathematics fractional integral operators also get importance for their use in the fractional control theory.

Magnus Gösta Mittag-Leffler introduced a function which is the natural extensions of many functions like exponential, hyperbolic and trigonometric etc. This function is well-known as the Mittag-Leffler function and is used very frequently in fractional calculus. Solutions of fractional differential equations are represented in the form of Mittag-Leffler functions. Many researchers have been published its numerous extensions and generalizations of various types. They also studied many integral transformations and proved the relation of Mitagg-Leffler function with some other functions (see, refs. [1-8]).

Special functions including gamma function, beta function, Mittag-Leffler function, hypergeometric function, Wright function are very important in the study of geometric function theory, applied mathematics, physics, statistics and many other subjects. The gamma function plays an important role in the formulation and representation of these functions. The extensions of Mittag-Leffler function is an interesting topic for researchers in which the classical notions linked with predefined Mittag-Leffler functions are investigated in more general prospect, see [9-11]. The Wright function is the generalization of hypergeometric function and several other special functions based on the gamma function, see [12-15]. The 
extensions of Mittag-Leffler function which are due to the gamma function can be obtained from the Wright function. The extended Mittag-Leffler function (5) so called the unified Mittag-Leffler function consists on generalized $p$-beta function and linked with several well-known predefined definitions in the literature. It will be interesting to investigate the unified Mittag-Leffler function in the form of other well-known functions.

Motivated and inspired by the ongoing research, the aim of this paper is to study the unified Mittag-Leffler function recently introduced by Zhang et al. [16] in the prospect of Wright generalized hypergeometric function. We will investigate this unified Mittag-Leffler function in the form of different well-known special functions. We also provide formulas of transformations like Beta, Laplace, Mellin and Whittaker in the form of Wright generalized hypergeometric function.

The classical Riemann-Liouville fractional integral operator is defined as follows:

Definition 1 ([17]). Let $f \in L[a, b]$. Then left-sided and right-sided Riemann-Liouville fractional integrals of a function $f$ of order $\beta$ where $\Re(\beta)>0$ are given by

$$
\begin{aligned}
& I_{a^{+}}^{\beta} f(x)=\frac{1}{\Gamma(\beta)} \int_{a}^{x}(x-t)^{\beta-1} f(t) d t, \quad x>a, \\
& I_{b^{-}}^{\beta} f(x)=\frac{1}{\Gamma(\beta)} \int_{x}^{b}(t-x)^{\beta-1} f(t) d t, \quad x<b,
\end{aligned}
$$

where $\Re(\beta)$ is real part of $\beta$ and $\Gamma(\beta)=\int_{0}^{\infty} e^{-z} z^{\beta-1} d z$.

It is interesting to note that if $f$ is symmetric about $\frac{a+x}{2}$, then the left-sided RiemannLiouville fractional integral of function $f$ satisfies the formula $I_{a^{+}}^{\beta} f(x)=I_{x^{-}}^{\beta} f(a)$. On the other hand if $f$ is symmetric about $\frac{x+b}{2}$, then the right-sided Riemann-Liouville fractional integral of function $f$ satisfies the formula $I_{b^{-}}^{\beta} f(x)=I_{x^{+}}^{\beta} f(b)$.

Mittag-Leffler functions are frequently used to define the fractional integral operators. Next, we give generalized fractional integral operators containing Mittag-Leffler function as follows:

Definition 2 (see [16]). Let $f \in L_{1}[a, b]$. Then $\forall \xi \in[a, b]$, the fractional integral operator with $\underline{a}=\left(a_{1}, a_{2}, \ldots, a_{n}\right), \underline{b}=\left(b_{1}, b_{2}, \ldots, b_{n}\right), \underline{c}=\left(c_{1}, c_{2}, \ldots, c_{n}\right)$, where $a_{i}, b_{i}, c_{i}, \omega \in \mathbb{C} ; i=$ $1,2,3, \ldots, n$ such that $\Re\left(a_{i}\right), \Re\left(b_{i}\right), \Re\left(c_{i}\right)>0, \forall i$. Furthermore, let $\alpha, \beta, \gamma, \delta, \mu, v, \lambda, \rho, \theta, t \in$ $\mathbb{C}, \min \Re(\alpha), \Re(\beta), \Re(\gamma), \Re(\delta), \Re(\theta)>0$ and $k \in(0,1) \cup \mathbb{N}$ with $k+\Re(\rho)<\Re(\delta+v+\alpha)$, $\operatorname{Im}(\rho)=\operatorname{Im}(\delta+v+\alpha)$ is defined as follows:

$$
\begin{aligned}
& \left(I_{a^{+}, \alpha, \beta, \gamma, \delta, \mu, v}^{\omega, \lambda, \rho, \theta, k, \eta} f\right)(\xi ; \underline{a}, \underline{b}, \underline{c}, \tilde{p})=\int_{a}^{\xi}(\xi-t)^{\beta-1} M_{\alpha, \beta, \gamma, \delta, \mu, v}^{\lambda, \rho, k, \eta}\left(\omega(\xi-t)^{\alpha} ; \underline{a}, \underline{b}, \underline{c}, \tilde{p}\right) f(t) d t, \\
& \left(I_{b^{-}, \alpha, \beta, \gamma, \delta, \mu, v}^{\omega, \lambda, \rho, \theta, k, \eta} f\right)(\xi ; \underline{a}, \underline{b}, \underline{c}, \tilde{p})=\int_{\xi}^{b}(t-\xi)^{\beta-1} M_{\alpha, \beta, \gamma, \delta, \mu, v}^{\lambda, \rho, k, \eta}\left(\omega(t-\xi)^{\alpha} ; \underline{a}, \underline{b}, \underline{c}, \tilde{p}\right) f(t) d t,
\end{aligned}
$$

where

$$
M_{\alpha, \beta, \gamma, \delta, \mu, v}^{\lambda, \rho, \theta, k, \eta}(z ; \underline{a}, \underline{b}, \underline{c}, \tilde{p})=\sum_{l=0}^{\infty} \frac{\prod_{i=1}^{n} \beta_{\tilde{p}}\left(b_{i}, a_{i}\right)(\lambda)_{\rho l}(\theta)_{k l}}{\prod_{i=1}^{n} \beta\left(c_{i}, a_{i}\right)(\gamma)_{\delta l}(\mu)_{v l}} \frac{z^{l}}{\Gamma(\alpha l+\beta)},
$$

is the unified Mittag-Leffler function and $\beta_{\tilde{p}}$ is the extension of beta function defined as follows:

$$
\beta_{\tilde{p}}(x, y)=\int_{0}^{1} t^{x-1}(1-t)^{y-1} e^{-\left(\frac{\tilde{p}}{t(1-t)}\right)} d t
$$


By setting $a_{i}=l, \tilde{p}=0$ and $\Re(\rho)>0$ in Definition 2, we get the fractional integral operator associated with generalized $Q$ function as follows (see [18]):

$$
\begin{aligned}
& \left(Q_{a^{+}, \alpha, \beta, \gamma, \delta, \mu, v}^{\omega, \lambda} f\right)(\xi ; \underline{b}, \underline{c})=\int_{a}^{\xi}(\xi-t)^{\beta-1} Q_{\alpha, \beta, \gamma, \gamma, \delta, \mu, v}^{\lambda, p, k, \eta}\left(\omega(\xi-t)^{\alpha} ; \underline{b}, \underline{c}\right) f(t) d t, \\
& \left(Q I_{b^{-}, \alpha, \beta, \beta, \gamma, \delta, \mu, v}^{\omega, \lambda, \theta, r, \eta} f\right)(\xi ; \underline{b}, \underline{c})=\int_{\tilde{\xi}}^{b}(t-\xi)^{\beta-1} Q_{\alpha, \beta, \gamma, \delta, \mu, v}^{\lambda, \rho, k, \eta}\left(\omega(t-\xi)^{\alpha} ; \underline{b}, \underline{c}\right) f(t) d t,
\end{aligned}
$$

where

$$
\left.Q_{\alpha, \beta, \gamma, \delta, \mu, \nu}^{\lambda, \rho, \theta, k, \eta}, \underline{a}\right)=\sum_{l=0}^{\infty} \frac{\prod_{i=1}^{n} \beta_{\tilde{p}}\left(b_{i}, l\right)(\lambda)_{\rho l}(\theta)_{k l} z^{l}}{\prod_{i=1}^{n} \beta\left(a_{i}, l\right)(\gamma)_{\delta l}(\mu)_{v l} \Gamma(\alpha l+\beta)},
$$

is a generalized $Q$ function defined in [19].

\section{Remark 1.}

(i) For $n=1, \rho=v=0, b_{1}=c_{1}+l k, a_{1}=\theta-\lambda, c_{1}=\lambda, \delta>0$, the Definition 2 reduces to ([20], Definition 2.1).

(ii) For $n=1, \rho=v=\tilde{p}=0, b_{1}=c_{1}+l k, a_{1}=\theta-\lambda, c_{1}=\lambda, \delta>0$, the Definition 2 reduces to ([4], Equation (8)).

(iii) For $\gamma=\delta=n=1, \rho=v=0, b_{1}=c_{1}+l k, a_{1}=\theta-\lambda, c_{1}=\lambda, \delta>0$, the Definition 2 reduces to ([3], Definition 2.3).

(iv) For $n=\gamma=\lambda=1, \rho=v=\tilde{p}=\omega=0, b_{1}=c_{1}+l k, a_{1}=\theta-\lambda, c_{1}=\lambda, \delta>0$, the Definition 2 gives Definition 1.

It is interesting to note that $\beta_{\tilde{p}}(x, y)=\beta_{\tilde{p}}(y, x)$ that is the function $\beta_{\tilde{p}}(x, y)$ is symmetric function in variables $x$ and $y$. Therefore we have

$$
M_{\alpha, \beta, \gamma, \delta, \mu, v}^{\lambda, \rho, \theta, k, \eta}(z ; \underline{a}, \underline{b}, \underline{p})=M_{\alpha, \beta, \gamma, \delta, \mu, v}^{\lambda, \rho, \theta, k, \eta}(z ; \underline{a}, \underline{a}, \tilde{p}),
$$

and

$$
\left(I_{a^{+}, \alpha, \beta, \gamma, \delta, \mu, v, v}^{\omega, \lambda} f\right)(\tilde{\xi} ; \underline{a}, \underline{b}, \underline{b}, \tilde{p})=\left(I_{a^{+}, \alpha, \beta, \gamma, \gamma, \delta, \mu, v}^{\omega, \lambda} f\right)(\tilde{\xi} ; \underline{b}, \underline{a}, \underline{a}, \tilde{p}) .
$$

Further, one can note that if $f$ is symmetric about $\frac{a+\xi}{2}$, then we have

$$
\left(I_{a^{+}, \alpha, \beta, \gamma, \delta, \mu, \nu, v}^{\omega, \lambda} f\right)(\tilde{\xi} ; \underline{a}, \underline{b}, \underline{c}, \tilde{p})=\left(I_{\mathcal{\zeta}^{-}, \alpha, \beta, \gamma, \gamma, \delta, \mu, v}^{\omega, \lambda} f\right)(a ; \underline{a}, \underline{b}, \underline{c}, \tilde{p}),
$$

and

$$
\left(I_{b^{-}, \alpha, \beta, \gamma, \gamma, \delta, \mu, v}^{\omega, \lambda} f\right)(\tilde{\xi} ; \underline{a}, \underline{b}, \underline{\mathcal{c}}, \tilde{p})=\left(I_{\mathcal{\zeta}^{-}, \alpha, \beta, \gamma, \gamma, \delta, \mu, v}^{\omega, \lambda} f\right)(b ; \underline{a}, \underline{b}, \underline{c}, \tilde{p}) .
$$

We will investigate some well-known transformations for the unified Mittag-Leffler function. Next, we recall these transformations as follows:

Definition 3 ([21]). Laplace transform of an integrable function $f$ on $[0, \infty)$ is defined as follows:

$$
F(s)=L(f(t))=\int_{0}^{\infty} e^{-s t} f(t) d t,
$$

where $s \in \mathbb{C}$ is the variable of the transform.

Definition 4 ([21]). The Euler beta transform of a function $f$ is defined by the following definite integral:

$$
\beta(f(z) ; a, b)=\int_{0}^{1} z^{a-1}(1-z)^{b-1} f(z) d z
$$


where $a$ and $b$ are any complex numbers with $\Re(a)>0, \Re(b)>0$.

Definition 5 ([21]). The Mellin transform of a function $f(z)$ is defined by following integral:

$$
\mathcal{M}(f(z) ; s)=f^{*}(s)=\int_{0}^{1} z^{s-1} f(z) d z, \Re(s)>0,
$$

and the inverse Mellin transform is given by

$$
f(z)=\mathcal{M}^{-1}\left(f^{*}(s) ; z\right)=\frac{1}{2 \pi \iota} \int_{c-\iota}^{c+\iota} z^{-s} f^{*}(s) d s, \quad c \in \mathbb{R} \text {. }
$$

Definition 6 ([22]). The Whittaker transform is defined by the following improper integral:

$$
\int_{0}^{\infty} e^{-\frac{t}{2}} t^{v-1} W_{\lambda, u}(t) d t=\int_{0}^{1} e^{-\frac{1}{2} v} v^{\xi+\eta l-1} W_{\lambda, u}(t) d t
$$

where $\Re(u+v)>-1 / 2$ and $W_{\lambda, u}(t)$ is the Whittaker confluent hypergeometric function.

Definition 7 ([6]). The Wright generalized hypergeometric function is defined as follows:

$$
{ }_{p} \Psi_{q}\left[\begin{array}{c}
\left(a_{1}, A_{1}\right), \ldots,\left(a_{p}, A_{q}\right) ; z \\
\left(b_{1}, B_{1}\right), \ldots,\left(b_{p}, B_{q}\right)
\end{array} z=\sum_{n=0}^{\infty} \frac{\prod_{i=1}^{p} \Gamma\left(a_{i}+A_{i} n\right)}{\prod_{i=1}^{q} \Gamma\left(b_{i}+B_{i} n\right)} \frac{z^{n}}{n !} .\right.
$$

The Wright generalized hypergeometric function can be represented in terms of Mellin-Barens type integral as follows (see, [23]):

$$
{ }_{p} \Psi_{q}\left[\begin{array}{c}
\left(a_{1}, A_{1}\right), \ldots,\left(a_{p}, A_{q}\right) \\
\left(b_{1}, B_{1}\right), \ldots,\left(b_{p}, B_{q}\right)
\end{array} z\right]=\frac{1}{2 \pi \iota} \int_{L} \Gamma(s) \frac{\prod_{i=1}^{p} \Gamma\left(a_{i}-A_{i} s\right)}{\prod_{j=1}^{q} \Gamma\left(b_{j}-B_{j} s\right)}(-z)^{-s} d s,
$$

where $L$ is the specially chosen contour $L$.

Definition 8 ([24]). The generalized hypergeometric function is defined as follows:

$$
{ }_{p} F_{q}\left[\begin{array}{l}
\alpha_{1}, \ldots, \alpha_{p} ; z \\
\beta_{1}, \ldots, \beta_{p}
\end{array}\right]=\sum_{n=0}^{\infty} \frac{\prod_{i=1}^{p}\left(a_{i}\right)_{n}}{\prod_{i=1}^{q}\left(b_{i}\right)_{n}} \frac{z^{n}}{n !}
$$

Definition 9 ([7]). The Fox's H-function is defined as follows:

$$
H_{p, q}^{m, n}\left[z \mid \begin{array}{l}
\left(a_{1}, \alpha_{1}\right), \ldots,\left(a_{p}, \alpha_{p}\right) \\
\left(b_{1}, \beta_{1}\right), \ldots,\left(b_{p}, \beta_{p}\right)
\end{array}\right]=\frac{1}{2 \pi \iota} \int_{L} \frac{\prod_{j=1}^{m} \Gamma\left(b_{j}+\beta_{j} s\right) \prod_{j=1}^{n} \Gamma\left(1-a_{j}+\alpha_{j} s\right)}{\prod_{j=m+1}^{q} \Gamma\left(1-b_{j}+\beta_{j} s\right) \prod_{j=n+1}^{p} \Gamma\left(a_{j}+\alpha_{j} s\right)} z^{-s} d s .
$$

Definition 10 ([24]). Generalized Laguerre or Sonine polynomials are defined as follows:

$$
L_{n}^{\alpha}(x)=\frac{(1+\alpha)_{n}}{n !}{ }_{1} F_{1}[-n ; 1+\alpha ; x]
$$

For a detailed study on hypergeometric functions and their applications, we refer the readers to $[6,15,24,25]$. In the upcoming section, we obtain relationship of the unified Mittag-Leffler function (5) with some known special functions. For this function we investigate integral transforms like beta, Laplace, Mellin, Whittaker in terms of Wright generalized hypergeometric function. We also find a recurrence relation along with another important and useful result.

\section{Main Results}

We formulate the unified Mittag-Leffler function (5) in the form of some well known special functions. 


\subsection{Relationship of $M_{\alpha, \beta, \gamma, \delta, \mu, v}^{\lambda, \rho, \theta, k, \eta}(z, \underline{b}, \underline{c}, \tilde{p})$ with Some Known Special Functions}

(1) Relationship with the Wright generalized hypergeometric function: By definition of the unified Mittag-Leffler function, we have

$$
\begin{aligned}
& M_{\alpha, \beta, \gamma, \delta, \mu, v}^{\lambda, \rho, \theta, k, \eta}(z ; \underline{a}, \underline{b}, \underline{p})=\sum_{l=0}^{\infty} \frac{\prod_{i=1}^{n} \beta_{\tilde{p}}\left(b_{i}, a_{i}\right)(\lambda)_{\rho l}(\theta)_{k l}}{\prod_{i=1}^{n} \beta\left(c_{i}, a_{i}\right)(\gamma)_{\delta l}(\mu)_{v l}} \frac{z^{l}}{\Gamma(\alpha l+\beta)} \\
& =\frac{\Gamma(\gamma) \Gamma(\mu) \prod_{i=1}^{n} \beta_{\tilde{p}}\left(b_{i}, a_{i}\right)}{\Gamma(\theta) \Gamma(\lambda) \prod_{i=1}^{n} \beta\left(c_{i}, a_{i}\right)}\left(\sum_{l=0}^{\infty} \frac{\Gamma(\lambda+\rho l) \Gamma(\theta+k l)(1)_{l} z^{l}}{\Gamma(\gamma+\delta l) \Gamma(\mu+v l) \Gamma(\alpha l+\beta) l !}\right) .
\end{aligned}
$$

By applying the Definition 7, we can write the above expression in terms of Wright generalized hypergeometric function as follows:

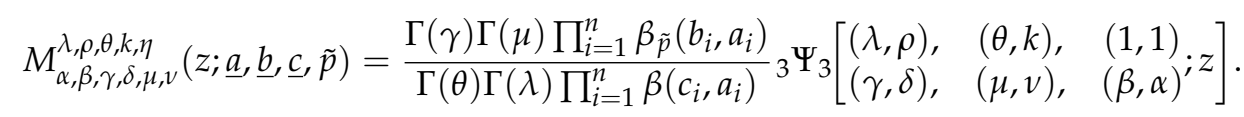

(2) Relationship with the generalized hypergeometric function: The relationship of the unified Mittag-Leffler function with the generalized hypergeometric function is given in the following theorem.

Theorem 1. Let $m \in \mathbb{N}$. Then $M_{\alpha, \beta, \gamma, \delta, \mu, v}^{\lambda, \rho, \theta, k, \eta}(z, \underline{a}, \underline{c}, \tilde{p})$ can be written in terms of generalized hypergeometric function as follows:

$$
\begin{gathered}
M_{m, \beta, \gamma, \delta, \mu, v}^{\lambda, \rho, \theta, k, \eta}(z ; \underline{a}, \underline{b}, \underline{c}, \tilde{p})=\frac{\prod_{i=1}^{n} \beta_{\tilde{p}}\left(b_{i}, a_{i}\right)}{\Gamma(\beta) \prod_{i=1}^{n} \beta\left(c_{i}, a_{i}\right)} \rho+k+1 \\
\text { where } \triangle(m, n)=\frac{n}{m}, \frac{n+1}{m}, \ldots, \frac{n+m-1}{m} .
\end{gathered}
$$

Proof. From (5) one can have

$$
\left.M_{\alpha, \beta, \gamma, \delta, \mu, v}^{\lambda, \rho, \theta, k, \eta}, \underline{a}, \underline{b}, \tilde{p}\right)=\frac{\prod_{i=1}^{n} \beta_{\tilde{p}}\left(b_{i}, a_{i}\right)}{\Gamma(\beta) \prod_{i=1}^{n} \beta\left(c_{i}, a_{i}\right)}\left(\sum_{l=0}^{\infty} \frac{(\lambda)_{\rho l}(\theta)_{k l} z^{l}}{(\gamma)_{\delta l}(\mu)_{v l}(\beta)_{\alpha l}}\right) .
$$

By setting $\alpha=m \in \mathbb{N}$, one can get

$$
\begin{aligned}
& M_{m, \beta, \gamma, \delta, \mu, v}^{\lambda, \rho, \theta, k, \eta}(z ; \underline{a}, \underline{b}, \underline{\mathcal{c}}, \tilde{p}) \\
& =\frac{\prod_{i=1}^{n} \beta_{\tilde{p}}\left(b_{i}, a_{i}\right)}{\Gamma(\beta) \prod_{i=1}^{n} \beta\left(c_{i}, a_{i}\right)} \sum_{l=0}^{\infty} \frac{\rho^{\rho l} \prod_{r=1}^{\rho}\left(\frac{\lambda+r-1}{\rho}\right)_{l} k^{k l} \prod_{j=1}^{k}\left(\frac{\theta+j-1}{k}\right)_{l}(1)_{l} z^{l}}{\delta^{\delta l} \prod_{d=1}^{\delta}\left(\frac{\gamma+d-1}{\delta}\right)_{l} v^{v l} \prod_{d=1}^{\delta}\left(\frac{\mu+e-1}{v}\right)_{l} m^{m l} \prod_{d=1}^{\delta}\left(\frac{\beta+f-1}{m}\right)_{l}} \\
& =\frac{\prod_{i=1}^{n} \beta_{\tilde{p}}\left(b_{i}, a_{i}\right)}{\Gamma(\beta) \prod_{i=1}^{n} \beta\left(c_{i}, a_{i}\right)} \rho+k+1 F_{\delta+v+m}\left[\begin{array}{ccc}
\triangle(\rho, \lambda), & \triangle(k, \theta), & 1 \\
\triangle(\delta, \gamma), & \triangle(v, \mu), & (m, \beta)^{\prime}
\end{array}\right] .
\end{aligned}
$$

Remark 2. (i) For $n=1, \rho=v=\tilde{p}=0, b_{1}=c_{1}+l k, a_{1}=\theta-\lambda, c_{1}=\lambda, \delta>0$ in (14), we get ([4], Theorem 3.1).

(ii) For $\gamma=\delta=n=1, \rho=v=0, b_{1}=c_{1}+l k, a_{1}=\theta-\lambda, c_{1}=\lambda, \delta>0$ (14), we get ([3], Theorem 3.2).

(3) Relationship with the Fox's H-function: In order to write $M_{\alpha, \beta, \gamma, \delta, \mu, v}^{\lambda, \rho, \theta, k, \eta}(z ; \underline{a}, \underline{b}, \underline{\mathcal{c}}, \tilde{p})$ in terms of Fox's H-function, we first express it as Mellin-Barnes-type integral. 
Theorem 2. The unified Mittag-Leffler function (5) can be expressed in terms of Mellin-Barnestype integral as follows:

$$
\begin{aligned}
M_{\alpha, \beta, \gamma, \delta, \mu, v}^{\lambda, \rho, \theta, k, \eta}(z ; \underline{a}, \underline{b}, \underline{c}, \tilde{p}) & =\frac{\Gamma(\gamma) \Gamma(\mu) \prod_{i=1}^{n} \beta_{\tilde{p}}\left(b_{i}, a_{i}\right)}{2 \pi \iota \Gamma(\theta) \Gamma(\lambda) \prod_{i=1}^{n} \beta\left(c_{i}, a_{i}\right)} \\
& \times \int_{L} \frac{\Gamma(s) \Gamma(1-s) \Gamma(\theta-k s) \Gamma(\lambda-\rho s)(-z)^{-s}}{\Gamma(\gamma-\delta s) \Gamma(\mu-v s) \Gamma(\beta-\alpha s)} d s,
\end{aligned}
$$

where $|\arg (z)|<\pi$; the contour of integration begins at $-1 \infty$ and ending at $1 \infty$, and intended to separate the poles of the integrand at $s=-n$ for all $n \in \mathbb{N}$ (to the left) from those at $s=n+1$ and at $s=\frac{\theta+\lambda+n}{k+\rho}$ for all $n \in \mathbb{R} \cup\{0\}$ (to the right).

Proof. In (13) writing the Wright generalized function in terms of Mellin-Barnes integral by using (11), one can have (15).

Hence one can have the following relation:

$$
\begin{aligned}
M_{\alpha, \beta, \gamma, \delta, \mu, v}^{\lambda, \rho, \theta j, \underline{\eta}}(z, \underline{b}, \underline{c}, \tilde{p})= & \frac{\Gamma(\gamma) \Gamma(\mu) \prod_{i=1}^{n} \beta_{\tilde{p}}\left(b_{i}, a_{i}\right)}{2 \pi \nu \Gamma(\theta) \Gamma(\lambda) \prod_{i=1}^{n} \beta\left(c_{i}, a_{i}\right)} \\
& \times \int_{L} \frac{\Gamma(s) \Gamma(1-s) \Gamma(\theta-k s) \Gamma(\lambda-\rho s)(-z)^{-s}}{\Gamma(\gamma-\delta s) \Gamma(\mu-v s) \Gamma(\beta-\alpha s)} d s \\
& =\frac{\Gamma(\gamma) \Gamma(\mu) \prod_{i=1}^{n} \beta_{\tilde{p}}\left(b_{i}, a_{i}\right)}{2 \pi i \Gamma(\theta) \Gamma(\lambda) \prod_{i=1}^{n} \beta\left(c_{i}, a_{i}\right)} \\
& \times H_{3,4}^{1,3}\left[-z \mid \begin{array}{ll}
(0,1), \quad(1-\theta, k), & (1-\lambda, \rho) \\
(0,1), \quad(1-\gamma, \delta), & (1-\mu, v), \quad(1-\beta, \alpha)
\end{array}\right] .
\end{aligned}
$$

The last equation is obtained by applying the Definition 9. This shows the representation of the unified Mittag-Leffler function (5) in terms of Fox's H-function.

(4) Relationship with generalized Laguerre polynomials:

By putting $\alpha=p, \beta=\xi+1, \lambda=-m, k=0, \delta=v=1$ and replace $z$ by $z^{p}, \rho$ by $q$, with $q \mid m q \in \mathbb{N}$ in (5), we get

$$
\begin{aligned}
& M_{p, \xi+1, \gamma, 1, \mu, 1}^{-m, q, 0, \eta}\left(z^{p} ; \underline{a}, \underline{b}, \underline{c}, \tilde{p}\right)=\sum_{l=0}^{\frac{m}{q}} \frac{\prod_{i=1}^{n} \beta_{\tilde{p}}\left(b_{i}, a_{i}\right)(-m)_{q l}}{\prod_{i=1}^{n} \beta\left(c_{i}, a_{i}\right)(\gamma)_{l}(\mu)_{l}} \frac{z^{p l}}{\Gamma(p l+\xi+1)} \\
& =\frac{\prod_{i=1}^{n} \beta_{\tilde{p}}\left(b_{i}, a_{i}\right)}{\prod_{i=1}^{n} \beta\left(c_{i}, a_{i}\right)} \sum_{l=0}^{\frac{m}{q}} \frac{(-1)^{q l} m ! z^{p l}}{(m-q l) !(\gamma)_{l}(\mu)_{l} \Gamma(p l+\xi+1)} \\
& =\frac{\prod_{i=1}^{n} \beta_{\tilde{p}}\left(b_{i}, a_{i}\right) \Gamma(m+1)}{\prod_{i=1}^{n} \beta\left(c_{i}, a_{i}\right) \Gamma(p m+\xi+1)} \sum_{l=0}^{\frac{m}{q}} \frac{(-1)^{q l} z^{p l} \Gamma(p m+\xi+1)}{(m-q l) !(\gamma)_{l}(\mu)_{l} \Gamma(p l+\xi+1)} \\
& =\frac{\prod_{i=1}^{n} \beta_{\tilde{p}}\left(b_{i}, a_{i}\right) \Gamma(m+1)}{\prod_{i=1}^{n} \beta\left(c_{i}, a_{i}\right) \Gamma(p m+\xi+1)} Z_{\frac{m}{q}, \gamma, \mu}^{\xi}(z, p),
\end{aligned}
$$

where $Z_{\frac{m}{q}, \gamma, \mu}^{\tau}(z, p)$ is a generalization of $Z_{\frac{m}{q}, \gamma}^{\tau}(z, p)$ and $Z_{\frac{m}{q}}^{\tau}(z, p)$ given by $[1,11]$, respectively.

Note that $Z_{\frac{m}{q}}^{\tau}(z, p)$ is a polynomial of degree $\frac{m}{q}$ in $z^{p}$.

Further for $q=p=\gamma=\mu=1, Z_{m, 1,1}^{\tau}(z, p)=L_{m}^{\xi}(z)$ where $L_{m}^{\tau}(z)$ is a generalized Laguerre polynomial. So that

$$
M_{p, \tilde{\xi}+1,1, \mu, 1}^{-m, q, \eta}(z ; \underline{a}, \underline{b}, \underline{c}, \tilde{p})=\frac{\prod_{i=1}^{n} \beta_{\tilde{p}}\left(b_{i}, a_{i}\right) \Gamma(m+1)}{\prod_{i=1}^{n} \beta\left(c_{i}, a_{i}\right) \Gamma(p m+\xi+1)} L_{m}^{\tilde{s}}(z) .
$$




\subsection{Integral Transforms of $\left.M_{\alpha, \beta, \gamma, \delta, \mu, v}^{\lambda, \rho, \theta, k, \eta}, \underline{b}, \underline{c}, \tilde{p}\right)$}

In this section, we have shown the image of the unified Mittag-Leffler function (5) under Beta, Laplace, Mellin and Whittaker transforms in terms of Wright generalized hypergeometric function.

Theorem 3. The beta transform of the unified Mittag-Leffler function in terms of wright geometric function can be represented as follows:

$$
\begin{aligned}
\beta\left(M_{\alpha, \beta, \gamma, \delta, \mu, v}^{\lambda, \rho, \theta, k, \eta}\left(x z^{\sigma} ; \underline{a}, \underline{b}, \underline{c}, \tilde{p}\right)\right) & =\frac{\Gamma(\gamma) \Gamma(\mu) \prod_{i=1}^{n} \beta_{\tilde{p}}\left(b_{i}, a_{i}\right)}{\Gamma(\theta) \Gamma(\lambda) \prod_{i=1}^{n} \beta\left(c_{i}, a_{i}\right)} \\
& \times{ }_{4} \Psi_{4}\left[\begin{array}{lll}
(\lambda, \rho), \quad(\theta, k), \quad(p, u) & (1,1) \\
(\gamma, \delta), & (\mu, v), \quad(u+v, p) & (\beta, \alpha)
\end{array}\right] .
\end{aligned}
$$

Proof. By definition of the beta transform we have

$$
\begin{aligned}
& \beta\left(M_{\alpha, \beta, \gamma, \delta, \mu, v}^{\lambda, \rho, \theta, k, \eta}\left(x z^{\sigma} ; \underline{a}, \underline{b}, \underline{c}, \tilde{p}\right)\right)=\int_{0}^{1} z^{u-1}(1-z)^{v} \sum_{l=0}^{\infty} \frac{\prod_{i=1}^{n} \beta_{\tilde{p}}\left(b_{i}, a_{i}\right)(\lambda)_{\rho l}(\theta)_{k l}}{\prod_{i=1}^{n} \beta\left(c_{i}, a_{i}\right)(\gamma)_{\delta l}(\mu)_{v l}} \frac{x^{l} z^{\sigma l}}{\Gamma(\alpha l+\beta)} \\
& =\sum_{l=0}^{\infty} \frac{\prod_{i=1}^{n} \beta_{\tilde{p}}\left(b_{i}, a_{i}\right)(\lambda)_{\rho l}(\theta)_{k l} x^{l}}{\prod_{i=1}^{n} \beta\left(c_{i}, a_{i}\right)(\gamma)_{\delta l}(\mu)_{v l} \Gamma(\alpha l+\beta)} \int_{0}^{1} z^{u+\sigma l-1}(1-z)^{v-1} d v \\
& =\sum_{l=0}^{\infty} \frac{\prod_{i=1}^{n} \beta_{\tilde{p}}\left(b_{i}, a_{i}\right)(\lambda)_{\rho l}(\theta)_{k l} x^{l}}{\prod_{i=1}^{n} \beta\left(c_{i}, a_{i}\right)(\gamma)_{\delta l}(\mu)_{v l} \Gamma(\alpha l+\beta)} \beta(u+\sigma l, v) \\
& =\frac{\prod_{i=1}^{n} \beta_{\tilde{p}}\left(b_{i}, a_{i}\right) \Gamma(v) \Gamma(\mu) \Gamma(\gamma)}{\prod_{i=1}^{n} \beta\left(c_{i}, a_{i}\right) \Gamma(\lambda) \Gamma(\theta)} \sum_{l=0}^{\infty} \frac{\Gamma(\lambda+\rho l) \Gamma(\theta+k l) \Gamma(u+\sigma l) x^{l}}{\Gamma(\gamma+\delta l) \Gamma(\mu+v l) \Gamma(\alpha l+\beta) \Gamma(u+\sigma l+v)} .
\end{aligned}
$$

By using the definition of Wright generalized hypergeometric function, one can obtain required equality.

Theorem 4. The Laplace transform of the unified Mittag-Leffler function in terms of Wright generalized hypergeometric function can be represented as follows:

$$
\begin{aligned}
L\left(z^{q-1} M_{\alpha, \beta, \gamma, \delta, \mu, v}^{\lambda, \rho, \theta, k, \eta}\left(x z^{\sigma} ; \underline{a}, \underline{b}, \underline{c}, \tilde{p}\right)\right) & =\frac{s^{-q} \Gamma(\gamma) \Gamma(\mu) \prod_{i=1}^{n} \beta_{\tilde{p}}\left(b_{i}, a_{i}\right)}{\Gamma(\theta) \Gamma(\lambda) \prod_{i=1}^{n} \beta\left(c_{i}, a_{i}\right)} \\
& \times_{4} \Psi_{3}\left[\begin{array}{lll}
(\lambda, \rho), & (\theta, k), \quad(m, q) & (1,1) ; \frac{x}{s^{\sigma}}
\end{array}\right] .
\end{aligned}
$$

Proof. By definition of the Laplace transform we have

$$
\begin{aligned}
& L\left(z^{q-1} M_{\alpha, \beta, \gamma, \gamma, \delta, \mu, v}^{\lambda, p, \theta, k, \eta}\left(x z^{\sigma} ; \underline{a}, \underline{b}, \underline{c}, \tilde{p}\right)\right)=\int_{0}^{1} z^{q-1} e^{-s z} M_{\alpha, \beta, \gamma, \delta, \mu, v}^{\lambda, \rho, \theta, k, \eta}\left(x z^{\sigma} ; \underline{a}, \underline{b}, \underline{c}, \tilde{p}\right) \\
& =\int_{0}^{1} z^{q-1} e^{-s z} \sum_{l=0}^{\infty} \frac{\prod_{i=1}^{n} \beta_{\tilde{p}}\left(b_{i}, a_{i}\right)(\lambda)_{\rho l}(\theta)_{k l}}{\prod_{i=1}^{n} \beta\left(c_{i}, a_{i}\right)(\gamma)_{\delta l}(\mu)_{v l}} \frac{x^{l} z^{\sigma l}}{\Gamma(\alpha l+\beta)} \\
& =\sum_{l=0}^{\infty} \frac{\prod_{i=1}^{n} \beta_{\tilde{p}}\left(b_{i}, a_{i}\right)(\lambda)_{\rho l}(\theta)_{k l} x^{l}}{\prod_{i=1}^{n} \beta\left(c_{i}, a_{i}\right)(\gamma)_{\delta l}(\mu)_{v l} \Gamma(\alpha l+\beta)} \int_{0}^{1} z^{q+\sigma l-1} e^{-s z} d z \\
& =\sum_{l=0}^{\infty} \frac{\prod_{i=1}^{n} \beta_{\tilde{p}}\left(b_{i}, a_{i}\right)(\lambda)_{\rho l}(\theta)_{k l} x^{l}}{\prod_{i=1}^{n} \beta\left(c_{i}, a_{i}\right)(\gamma)_{\delta l}(\mu)_{v l} \Gamma(\alpha l+\beta)} \frac{\Gamma(q+\sigma l)}{s^{q+\sigma l}} \\
& =\frac{\prod_{i=1}^{n} \beta_{\tilde{p}}\left(b_{i}, a_{i}\right) s^{-q} \Gamma(v) \Gamma(\mu) \Gamma(\gamma)}{\prod_{i=1}^{n} \beta\left(c_{i}, a_{i}\right) \Gamma(\lambda) \Gamma(\theta)} \sum_{l=0}^{\infty} \frac{\Gamma(\lambda+\rho l) \Gamma(\theta+k l) \Gamma(q+\sigma l)(1)_{l} x^{l}}{\Gamma(\gamma+\delta l) \Gamma(\mu+v l) \Gamma(\alpha l+\beta)^{\sigma l} l !} .
\end{aligned}
$$

By using the definition of wright geometric function, one can obtain required equality. 
Theorem 5. The Mellin transform of the unified Mittag-Leffler function in terms of Wright geometric function can be represented as follows:

$$
\begin{aligned}
\mathcal{M}\left(M_{\alpha, \beta, \gamma, \delta, \mu, v}^{\lambda, \rho, \theta, k, \eta}(-\omega z ; \underline{a}, \underline{b}, \underline{c}, \tilde{p}) ; s\right) & =\frac{\Gamma(\gamma) \Gamma(\mu) \prod_{i=1}^{n} \beta_{\tilde{p}}\left(b_{i}, a_{i}\right)}{\Gamma(\theta) \Gamma(\lambda) \prod_{i=1}^{n} \beta\left(c_{i}, a_{i}\right)} \\
& \times \frac{\Gamma(s) \Gamma(1-s) \Gamma(\theta-k s) \Gamma(\lambda-\rho s)(-z)^{-s}}{\Gamma(\gamma-\delta s) \Gamma(\mu-v s) \Gamma(\beta-\alpha s)} .
\end{aligned}
$$

Proof. According to Theorem 2, we can write

$$
\begin{aligned}
M_{\alpha, \beta, \gamma, \delta, \mu, v}^{\lambda, \rho, \theta, k, \underline{u}}(z, \underline{b}, \underline{c}, \tilde{p}) & =\frac{\Gamma(\gamma) \Gamma(\mu) \prod_{i=1}^{n} \beta_{\tilde{p}}\left(b_{i}, a_{i}\right)}{2 \pi \iota \Gamma(\theta) \Gamma(\lambda) \prod_{i=1}^{n} \beta\left(c_{i}, a_{i}\right)} \\
& \times \int_{L} \frac{\Gamma(s) \Gamma(1-s) \Gamma(\theta-k s) \Gamma(\lambda-\rho s)(-z)^{-s}}{\Gamma(\gamma-\delta s) \Gamma(\mu-v s) \Gamma(\beta-\alpha s)} d s,
\end{aligned}
$$

where $f^{*}(s)=\frac{\Gamma(s) \Gamma(1-s) \Gamma(\theta-k s) \Gamma(\lambda-\rho s)}{\Gamma(\gamma-\delta s) \Gamma(\mu-\nu s) \Gamma(\beta-\alpha s)}$ and $L$ is the contour of integration that begins at $c-\iota \infty$ and ends at $c+\iota \infty ; c \in \mathbb{R}$.

$$
M_{\alpha, \beta, \gamma, \delta, \mu, v}^{\lambda, \rho, \theta, k, \eta}(z ; \underline{a}, \underline{c}, \tilde{p})=\frac{\Gamma(\gamma) \Gamma(\mu) \prod_{i=1}^{n} \beta_{\tilde{p}}\left(b_{i}, a_{i}\right)}{\Gamma(\theta) \Gamma(\lambda) \prod_{i=1}^{n} \beta\left(c_{i}, a_{i}\right)} \mathcal{M}^{-1}\left(f^{*}(s) ; z\right) .
$$

By applying Mellin transform on both sides, we can obtain the required equality.

Theorem 6. The Whittaker transform of the unified Mittag-Leffler function in terms of Wright geometric function can be represented as follows:

$$
\begin{aligned}
& \int_{0}^{\infty} e^{-\frac{1}{2} \phi t} t^{\tilde{s}-1} W_{\lambda, u}(\phi t) M_{\alpha, \beta, \gamma, \delta, \mu, \nu}^{\lambda, \rho, \theta, k, \eta}\left(x z^{\sigma} ; \underline{a}, \underline{b}, \underline{c}, \tilde{p}\right) d t=\frac{\Gamma(\gamma) \Gamma(\mu) \phi^{-\tilde{\xi}} \prod_{i=1}^{n} \beta_{\tilde{p}}\left(b_{i}, a_{i}\right)}{\Gamma(\theta) \Gamma(\lambda) \prod_{i=1}^{n} \beta\left(c_{i}, a_{i}\right)}
\end{aligned}
$$

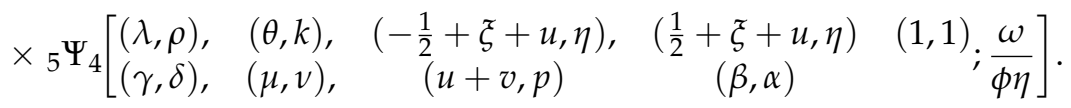

Proof. By definition of the Whittaker transform we have

$$
\begin{aligned}
& \int_{0}^{\infty} e^{-\frac{1}{2} \phi t} t^{\tilde{\xi}-1} W_{\lambda, u}(\phi t) M_{\alpha, \beta, \gamma, \delta, \mu, v}^{\lambda, \rho, \theta, k, \eta}\left(\omega t^{\eta} ; \underline{a}, \underline{b}, \underline{c}, \tilde{p}\right) d t \\
& =\int_{0}^{1} e^{-\frac{1}{2} \phi t} t^{\tilde{\xi}-1} W_{\lambda, u}(\phi t) \sum_{l=0}^{\infty} \frac{\prod_{i=1}^{n} \beta_{\tilde{p}}\left(b_{i}, a_{i}\right)(\lambda)_{\rho l}(\theta)_{k l}}{\prod_{i=1}^{n} \beta\left(c_{i}, a_{i}\right)(\gamma)_{\delta l}(\mu)_{v l}} \frac{\omega^{l} t^{\eta l}}{\Gamma(\alpha l+\beta)} .
\end{aligned}
$$

Setting $\phi t=v$, we get

$$
\begin{aligned}
& \int_{0}^{1} e^{-\frac{1}{2} \phi t} t^{\xi-1} W_{\lambda, u}(v) \sum_{l=0}^{\infty} \frac{\prod_{i=1}^{n} \beta_{\tilde{p}}\left(b_{i}, a_{i}\right)(\lambda)_{\rho l}(\theta)_{k l}}{\prod_{i=1}^{n} \beta\left(c_{i}, a_{i}\right)(\gamma)_{\delta l}(\mu)_{v l}} \frac{\omega^{l} t^{\eta l}}{\Gamma(\alpha l+\beta)} \\
& =\sum_{l=0}^{\infty} \frac{\prod_{i=1}^{n} \beta_{\tilde{p}}\left(b_{i}, a_{i}\right)(\lambda)_{\rho l} \phi^{-\xi}(\theta)_{k l}}{\prod_{i=1}^{n} \beta\left(c_{i}, a_{i}\right)(\gamma)_{\delta l}(\mu)_{v l} \Gamma(\alpha l+\beta)}\left(\frac{\omega}{\phi^{\eta}}\right)^{l} \int_{0}^{1} e^{-\frac{1}{2} v} v^{\xi+\eta l-1} W_{\lambda, u}(v) \\
& =\sum_{l=0}^{\infty} \frac{\prod_{i=1}^{n} \beta_{\tilde{p}}\left(b_{i}, a_{i}\right)(\lambda)_{\rho l}(\theta)_{k l}}{\prod_{i=1}^{n} \beta\left(c_{i}, a_{i}\right)(\gamma)_{\delta l}(\mu)_{v l} \Gamma(\alpha l+\beta)}\left(\frac{\omega}{\phi^{\eta}}\right)^{l} \Gamma\left(\frac{1}{2}+\xi+u, \eta\right) \Gamma\left(-\frac{1}{2}+\xi+u, \eta\right) \\
& =\frac{\prod_{i=1}^{n} \beta_{\tilde{p}}\left(b_{i}, a_{i}\right) \Gamma(v) \Gamma(\mu) \Gamma(\gamma)}{\prod_{i=1}^{n} \beta\left(c_{i}, a_{i}\right) \Gamma(\lambda) \Gamma(\theta)}\left(\frac{\omega}{\phi^{\eta}}\right)^{l} \\
& \times \sum_{l=0}^{\infty} \frac{\Gamma(\lambda+\rho l) \Gamma(\theta+k l) \Gamma(u+\sigma l) \Gamma\left(\frac{1}{2}+\xi+u, \eta\right) \Gamma\left(-\frac{1}{2}+\xi+u, \eta\right)}{\Gamma(\gamma+\delta l) \Gamma(\mu+v l) \Gamma(\alpha l+\beta) \Gamma(u+\sigma l+v) \Gamma(1-\lambda+\xi+\eta l)} .
\end{aligned}
$$


By using the definition of Wright generalized hypergeometric function, one can obtain required equality.

Next, we give the differential recurrence relation form of the unified Mittag-Leffler function.

Theorem 7. If $\underline{a}=\left(a_{1}, a_{2}, \ldots, a_{n}\right), \underline{b}=\left(b_{1}, b_{2}, \ldots, b_{n}\right), \underline{c}=\left(c_{1}, c_{2}, \ldots, c_{n}\right), \beta, \gamma, \delta, \mu$, $v, \lambda, \rho, \theta, a_{i}, b_{i}, c_{i} \in \mathbb{C}, \min \{\Re(\beta), \Re(\gamma), \Re(\delta), \Re(\theta)\}>0, \alpha>0$ and $k \in(0,1) \cup \mathbb{N}$ with $p \geq 0$, then the differential recurrence relation form is given as follows

$$
\beta M_{\alpha, \beta+1, \gamma, \delta, \mu, v}^{\lambda, \rho, k, \eta}(z ; \underline{a}, \underline{b}, \underline{c}, \tilde{p})+\alpha z \frac{d}{d z} M_{\alpha, \beta+1, \gamma, \delta, \mu, v}^{\lambda, \rho, k, \eta}(\underline{z}, \underline{b}, \underline{c}, \tilde{p})=M_{\alpha, \beta, \gamma, \delta, \mu, v}^{\lambda, \rho, k, \eta}(z ; \underline{a}, \underline{b}, \underline{c}, \tilde{p}) .
$$

Proof. By applying the definition we have

$$
\begin{aligned}
& M_{\alpha, \beta+1, \gamma, \delta, \mu, v}^{\lambda, \rho, k, \eta}(z ; \underline{a}, \underline{b}, \underline{c}, \tilde{p})+\alpha z M_{\alpha, \beta+1, \gamma, \delta, \mu, v}^{\lambda, \rho, k, \eta}(z ; \underline{a}, \underline{b}, \underline{c}, \tilde{p}) \\
& =\beta \sum_{l=0}^{\infty} \frac{\prod_{i=1}^{n} \beta_{\tilde{p}}\left(b_{i}, a_{i}\right)(\lambda)_{\rho l}(\theta)_{k l}}{\prod_{i=1}^{n} \beta\left(c_{i}, a_{i}\right)(\gamma)_{\delta l}(\mu)_{v l}} \frac{z^{l}}{\Gamma(\alpha l+\beta+1)}+\alpha z \frac{d}{d z} \sum_{l=0}^{\infty} \frac{\prod_{i=1}^{n} \beta_{\tilde{p}}\left(b_{i}, a_{i}\right)(\lambda)_{\rho l}(\theta)_{k l}}{\prod_{i=1}^{n} \beta\left(c_{i}, a_{i}\right)(\gamma)_{\delta l}(\mu)_{v l}} \frac{z^{l}}{\Gamma(\alpha l+\beta+1)} \\
& =\beta \sum_{l=0}^{\infty} \frac{\prod_{i=1}^{n} \beta_{\tilde{p}}\left(b_{i}, a_{i}\right)(\lambda)_{\rho l}(\theta)_{k l}}{\prod_{i=1}^{n} \beta\left(c_{i}, a_{i}\right)(\gamma)_{\delta l}(\mu)_{v l}} \frac{z^{l}}{\Gamma(\alpha l+\beta+1)}+\alpha z \sum_{l=0}^{\infty} \frac{\prod_{i=1}^{n} \beta_{\tilde{p}}\left(b_{i}, a_{i}\right)(\lambda)_{\rho l}(\theta)_{k l}}{\prod_{i=1}^{n} \beta\left(c_{i}, a_{i}\right)(\gamma)_{\delta l}(\mu)_{v l}} \frac{l z^{l-1}}{\Gamma(\alpha l+\beta+1)} \\
& =\beta \sum_{l=0}^{\infty} \frac{\prod_{i=1}^{n} \beta_{\tilde{p}}\left(b_{i}, a_{i}\right)(\lambda)_{\rho l}(\theta)_{k l}}{\prod_{i=1}^{n} \beta\left(c_{i}, a_{i}\right)(\gamma)_{\delta l}(\mu)_{v l}} \frac{z^{l}}{\Gamma(\alpha l+\beta+1)}+\alpha \sum_{l=0}^{\infty} \frac{\prod_{i=1}^{n} \beta_{\tilde{p}}\left(b_{i}, a_{i}\right)(\lambda)_{\rho l}(\theta)_{k l}}{\prod_{i=1}^{n} \beta\left(c_{i}, a_{i}\right)(\gamma)_{\delta l}(\mu)_{v l}} \frac{l z^{l}}{\Gamma(\alpha l+\beta+1)} \\
& =\sum_{l=0}^{\infty} \frac{\prod_{i=1}^{n} \beta_{\tilde{p}}\left(b_{i}, a_{i}\right)(\lambda)_{\rho l}(\theta)_{k l}}{\prod_{i=1}^{n} \beta\left(c_{i}, a_{i}\right)(\gamma)_{\delta l}(\mu)_{v l}} \frac{z^{l}(\alpha l+\beta)}{\Gamma(\alpha l+\beta+1)}=M_{\alpha, \beta, \gamma, j, \delta, \nu}^{\lambda, p}(z ; \underline{a}, \underline{b}, \underline{\mathcal{c}}, \tilde{p}) .
\end{aligned}
$$

Theorem 8. If $\underline{a}=\left(a_{1}, a_{2}, \ldots, a_{n}\right), \underline{b}=\left(b_{1}, b_{2}, \ldots, b_{n}\right), \underline{c}=\left(c_{1}, c_{2}, \ldots, c_{n}\right), \beta, \gamma, \delta, \mu, v$, $\lambda, \rho, \theta, a_{i}, b_{i}, c_{i} \in \mathbb{C}, \min \{\Re(\beta), \Re(\gamma), \Re(\delta), \Re(\theta)\}>0, \alpha>0$ and $k \in(0,1) \cup \mathbb{N}$ with $p \geq 0$, then for $f(t)=(t-a)^{q-1}$ it follows:

$$
\left(I_{a^{+}, \alpha, \beta, \gamma, \delta, \beta, \nu, v}^{\omega, \lambda}(t-a)^{q-1}\right)(\xi ; \underline{a}, \underline{b}, \underline{c}, \tilde{p})=(\xi-a)^{-1+q+\beta} \Gamma(q) M_{\alpha, \beta+q, \gamma, \delta, \delta, \mu, v}^{\lambda, \rho, k, \eta}\left(\omega(\xi-a)^{\alpha} ; \underline{a}, \underline{b}, \underline{c}, \tilde{p}\right) .
$$

Proof. By definition, we have

$$
\begin{aligned}
& \left(I_{a^{+}, \alpha, \beta, \beta, \gamma, \delta, \mu, v}^{\omega, \lambda}(t-a)^{q-1}\right)(\xi ; \underline{a}, \underline{b}, \underline{c}, \tilde{p}) \\
& =\int_{a}^{\xi}(\xi-t)^{\beta-1} M_{\alpha, \beta, \gamma, \delta, \mu, v}^{\lambda, \rho, k}\left(\omega(\xi-t)^{\alpha} ; \underline{a}, \underline{b}, \underline{c}, \tilde{p}\right)(t-a)^{q-1} d t \\
& =\int_{a}^{\xi}(\xi-t)^{\beta-1} \sum_{l=0}^{\infty} \frac{\prod_{i=1}^{n} \beta_{\tilde{p}}\left(b_{i}, a_{i}\right)(\lambda)_{\rho l}(\theta)_{k l}}{\prod_{i=1}^{n} \beta\left(c_{i}, a_{i}\right)(\gamma)_{\delta l}(\mu)_{v l}} \frac{\left(\omega(\xi-t)^{\alpha}\right)^{l}}{\Gamma(\alpha l+\beta)}(t-a)^{q-1} d t \\
& =\sum_{l=0}^{\infty} \frac{\prod_{i=1}^{n} \beta_{\tilde{p}}\left(b_{i}, a_{i}\right)(\lambda)_{\rho l}(\theta)_{k l}}{\prod_{i=1}^{n} \beta\left(c_{i}, a_{i}\right)(\gamma)_{\delta l}(\mu)_{v l}} \frac{\omega^{l}}{\Gamma(\alpha l+\beta)} \int_{a}^{\tilde{\zeta}}(\xi-t)^{\beta-1}(t-a)^{q-1} d t \\
& =(\xi-a)^{-1+q+\beta} \Gamma(q)\left(\sum_{l=0}^{\infty} \frac{\prod_{i=1}^{n} \beta_{\tilde{p}}\left(b_{i}, a_{i}\right)(\lambda)_{\rho l}(\theta)_{k l}}{\prod_{i=1}^{n} \beta\left(c_{i}, a_{i}\right)(\gamma)_{\delta l}(\mu)_{v l}} \frac{\omega^{l}(\xi-a)^{\alpha l}}{\Gamma(q+\alpha l+\beta)}\right) \\
& =(\xi-a)^{-1+q+\beta} \Gamma(q) M_{\alpha, \beta+q, \gamma, \gamma, \delta, \mu, v}^{\lambda, \rho, k, \eta}\left(\omega(\xi-a)^{\alpha} ; \underline{a}, \underline{b}, \underline{c}, \tilde{p}\right),
\end{aligned}
$$

where for integration, we have used Mathematica software

$$
\int_{a}^{\tau}(\xi-t)^{\beta-1}(t-a)^{q-1} d t=\frac{(\xi-a)^{-1+q+\alpha l+\beta} \Gamma(q) \Gamma(\alpha l+\beta)}{\Gamma(q+\alpha l+\beta)} .
$$


Setting $a=0$ and $\xi=1$, we get the next result:

Corollary 1. If $\underline{a}=\left(a_{1}, a_{2}, \ldots, a_{n}\right), \underline{b}=\left(b_{1}, b_{2}, \ldots, b_{n}\right), \underline{c}=\left(c_{1}, c_{2}, \ldots, c_{n}\right), \beta, \gamma, \delta, \mu, v, \lambda$, $\rho, \theta, a_{i}, b_{i}, c_{i} \in \mathbb{C}, \min \{\Re(\beta), \Re(\gamma), \Re(\delta), \Re(\theta)\}>0, \alpha>0$ and $k \in(0,1) \cup \mathbb{N}$ with $p \geq 0$, then

$$
\int_{0}^{1} t^{q-1}(1-t)^{\beta-1} M_{\alpha, \beta, \gamma, \delta, \mu, v}^{\lambda, \rho, k, \eta}\left(\omega(1-t)^{\alpha} ; \underline{a}, \underline{b}, \underline{c}, \tilde{p}\right) d t=\Gamma(q) M_{\alpha, \beta+q, \gamma, \delta, \mu, v}^{\lambda, \rho, k, \eta}(\omega ; \underline{a}, \underline{b}, \underline{c}, \tilde{p}) .
$$

Remark 3. (i) For $n=1, \rho=v=0, b_{1}=c_{1}+l k, a_{1}=\theta-\lambda, c_{1}=\lambda$ in (21), then ([20], Theorem 2.5) is obtained.

(ii) For $\gamma=\delta=n=1, \rho=v=0, b_{1}=c_{1}+l k, a_{1}=\theta-\lambda, c_{1}=\lambda, \delta>0$ (21), we get ([3], Theorem 3.1).

(iii) For $\gamma=\delta=n=1, \rho=v=\tilde{p}=0, b_{1}=c_{1}+l k, a_{1}=\theta-\lambda, c_{1}=\lambda, \delta>0$ (21), we get ([11], Theorem 2.4).

Corollary 2. By setting $a_{i}=l$ and $\tilde{p}=0$ in (21), we get the equation for the integral operator given in (7) as follows:

$$
\left.\left(Q_{a^{+}, \alpha, \beta, \gamma, \delta, j, \mu, v}^{\omega} I_{\lambda}, \rho, a\right)^{q-1}\right)(\xi ; \underline{b}, \underline{c})=(\xi-a)^{-1+q+\beta} \Gamma(q) Q_{\alpha, \beta+q, \gamma, \delta, \mu, v}^{\lambda, \rho, k, \eta}\left(\omega(\xi-a)^{\alpha} ; \underline{b}, \underline{c}, \tilde{p}\right) .
$$

Next, we give an example as follows:

Example 1. The function $(t-a)^{2}$ is defined on $[0,2 a]$ is symmetric about $a$. By using (9) we have

$$
\left(I_{a^{+}, \alpha, \beta, \gamma, \delta, j, \mu, v}^{\omega, \lambda, p}(t-a)^{2}\right)(\tilde{\xi} ; \underline{a}, \underline{b}, \underline{c}, \tilde{p})=\left(I_{\mathcal{\zeta}^{-}, \alpha, \beta, \gamma, \gamma, \delta, \mu, v}^{\omega, \lambda}(t-a)^{2}\right)(a ; \underline{a}, \underline{b}, \underline{c}, \tilde{p}) .
$$

By applying Theorem 8 for $q=3$ and using (24) one can easily see that

$$
\begin{aligned}
\left(I_{a^{+}, \alpha, \beta, \gamma, \delta, \delta, \mu, v}^{\omega, \lambda, \rho, \theta, k, \eta}(t-a)^{2}\right)(\xi ; \underline{a}, \underline{b}, \underline{c}, \tilde{p}) & =(\tilde{\xi}-a)^{2+\beta} \Gamma(3) M_{\alpha, \beta+3, \gamma, \delta, \mu, \nu}^{\lambda, \rho, k, \eta}\left(\omega(\tilde{\xi}-a)^{\alpha} ; \underline{a}, \underline{b}, \underline{c}, \tilde{p}\right) \\
& =\left(I_{\mathcal{\zeta}^{-}, \alpha, \beta, \gamma, \gamma, \delta, \mu, \nu}^{\omega, \lambda}(t-a)^{2}\right)(a ; \underline{a}, \underline{b}, \underline{c}, \tilde{p}) .
\end{aligned}
$$

\section{Conclusions}

In this study, we have given the relationship of generalized Mittag-Leffler function with well known special functions. Some integral transforms of the unified Mittag-Leffler function in terms of Wright generalized function were formulated. We also obtained differential recurrence relation. Some formulas of fractional integrals for symmetric functions were given. For future work, the unified Mittag-Leffler function can be studied in the prospect of geometric function theory. It can also be applied to study the generalizations of concepts directly linked with the classical Mittag-Leffler functions.

Author Contributions: Conceptualization, C.J., G.F., H.Y. and K.N.; Investigation, H.Y. and K.N.; Methodology, C.J.; Supervision, G.F.; Writing—original draft, H.Y.; Writing—review \& editing, C.J., G.F., H.Y. and K.N. All authors have read and agreed to the published version of the manuscript.

Funding: This research received no external funding.

Institutional Review Board Statement: Not applicable.

Informed Consent Statement: Not applicable.

Data Availability Statement: Not applicable.

Acknowledgments: This work was supported by development fund foundation, Gyeongsang National University, 2021 and the National Science, Research, and Innovation Fund (NSRF), Thailand.

Conflicts of Interest: The authors declare no conflict of interest. 


\section{References}

1. Khan, M.A.; Ahmed, S. On some properties of the generalized Mittag-Leffler function. SpringerPlus 2013, 2, 337. [CrossRef] [PubMed]

2. Prajapati, J.C.; Dave, B.I.; Nathwani, B.V. On a unification of generalized Mittag-Leffler function and family of Bessel functions. Adv. Pure Math. 2013, 3, 127-137. [CrossRef]

3. Rahman, G.; Baleanu, D.; Qurashi, M.A.; Purohit, S.D.; Mubeen, S.; Arshad, M. The extended Mittag-Leffler function via fractional calculus. J. Nonlinear Sci. Appl. 2017, 10, 4244-4253. [CrossRef]

4. Salim, T.O.; Faraj, A.W. A generalization of Mittag-Leffler function and integral operator associated with integral calculus. J. Frac. Calc. Appl. 2012, 3, 1-13.

5. Qi, F.; Nisar, K.S. Some integral transforms of the generalized k-Mittag-Leffler function. Publ. Inst. Math. 2019, 106, 125-133. [CrossRef]

6. Srivastava, H.M.; Manocha, H.L. A Treatise on Generating Functions; John Wiley and Sons: New York, NY, USA, 1984.

7. Gorenflo, R.; Kilbas, A.A.; Rogosin, S.V. On the generalized Mittag-Leffler type functions. Integral Transforms Spec. Funct. 1998, 7, 215-224. [CrossRef]

8. Bansal, M.K.; Kumar, D. On the integral operators pertaining to a family of incomplete I-functions. AIMS Math. 2020, 5, 1247-1259. [CrossRef]

9. Singh, P.; Jain, S.; Cattani, C. Some Unified Integrals for Generalized Mittag-Leffler Functions. Axioms 2021, 10, 261. [CrossRef]

10. Almalahi, M.A.; Ghanim, F.; Botmart, T.; Bazighifan, O.; Askar, S. Qualitative Analysis of Langevin Integro-Fractional Differential Equation under Mittag-Leffler Functions Power Law. Fractal Fract. 2021, 5, 266. [CrossRef]

11. Shukla, A.K.; Prajapati, J.C. On a generalization of Mittag-Leffler function and its properties. J. Math. Anal. Appl. 2007, 336, 797-811. [CrossRef]

12. Cătaş, A. On the Fekete-Szegö Problem for Meromorphic Functions Associated with $p$, q-Wright Type Hypergeometric Function. Symmetry 2021, 13, 2143. [CrossRef]

13. Naheed, S.; Mubeen, S.; Rahman, G.; Khan, Z.A.; Nisar, K.S. Certain Integral and Differential Formulas Involving the Product of Srivastava's Polynomials and Extended Wright Function. Fractal Fract. 2022, 6, 93. [CrossRef]

14. Apelblat, A.; González-Santander, J.L. The Integral Mittag-Leffler, Whittaker and Wright Functions. Mathematics 2021, 9, 3255. [CrossRef]

15. Srivastava, H.M.; Kumar, A.; Das, S.; Mehrez, K. Geometric properties of a certain class of Mittag-Leffler-type functions. Fractal Fract. 2020, 6, 54. [CrossRef]

16. Zhang, Y.; Farid, G.; Salleh, Z.; Ahmad, A. On a unified Mittag-Leffler function and associated fractional integral operator. Math. Probl. Eng. 2021, 2021, 6043769. [CrossRef]

17. Kilbas, A.A.; Srivastava, H.M.; Trujillo, J.J. Theory and Applications of Fractional Differential Equations; North-Holland Mathematics Studies, 204; Elsevier: New York, NY, USA; London, UK, 2006.

18. Zhou, S.S.; Farid, G.; Ahmad, A. Fractional versions of Minkowski-type integral inequalities via unified Mittag-Leffler function. Adv. Cont. Discr. Mod. 2022, 2022, 9. [CrossRef]

19. Bhatnagar, D.; Pandey, R.M. A study of some integral transforms on $Q$ function. South East Asian J. Math. Math. Sci. 2020, 16, 99-110.

20. Andrić, M.; Farid, G.; Pečarić, J. A further extension of Mittag-Leffler function. Fract. Calc. Appl. Anal. 2018, 21, 1377-1395. [CrossRef]

21. Sneddon, I.N. The Use of Integral Transforms; Tata McGraw Hill: New Delhi, India, 1979.

22. Wittaker, E.T.; Watson, G.N. A Course of Modern Analysis; Cambridge Univ. Press: Cambridge, UK, 1962.

23. Erdélyi, A.; Magnus, W.; Oberhettinger, F.; Tricomi, F.G. Higher Transcendental Tunctions; McGraw-Hill: New York, NY, USA; Toronto, ON, Canada; London, UK, 1953; Volume 1.

24. Rainville, E.D. Special Functions; Macmillan: New York, NY, USA, 1960.

25. Bansal, M.K.; Kumar, D.; Jain, R. A study of Marichev-Saigo-Maeda fractional integral operators associated with S-generalized Gauss hypergeometric function. Kyungpook Math. J. 2019, 59, 433-443. 